\title{
Gestión del conocimiento
}

y sistemas de calidad

en los clusters de empresas familiares

FECHA DE RECEPCIÓN: 10 de febrero de 2010 FECHA DE APROBACIÓN: 29 de abril de 2010 Pp. 70-85

\section{Knowledge \\ management and quality systems inn family company clusters}

\section{José Manuel Saiz Álvarez* Beatriz Olalla Caballero**}

*Doctor "cum laude" en Ciencias Empresariales, Universidad Autónoma de Madrid (España) y Doctor "cum laude" en Sociología, Universidad

Pontificia de Salamanca (España); profesor de la Universidad Antonio de Nebrija y de la Universidad Pontificia de Salamanca (Campus de Madrid)

** Desde Octubre 2008.- Tutor de la alumna de Doctorado en Ingeniería Informática, BeatrizOlalla Caballero, correspondiente al $2^{\circ}$ Curso de Doctorado, Facultad de Informática, Universidad Pontificia de Salamanca en Madrid. Alumna de Tecnologías de

Radiocomunicaciones III Programa que tiene el apoyo de Programa Europeo Europractice 
T as empresas familiares constituyen el núcleo empresarial de buena parte de los países Lcon independencia de su desarrollo. Dado el proceso de fuerte competencia fruto de la globalización económica, la formación de clusters y la gestión tanto del conocimiento como de los sistemas de calidad dentro de las corporaciones tiene una importancia fundamental para la supervivencia de la empresa a largo plazo. A lo largo de este trabajo se realiza un análisis de la interacción entre los clusters, la gestión del conocimiento y los sistemas de calidad de las empresas, en especial aplicado a la empresa familiar.

\section{ABSTRACT}

$F^{a}$ amily companies make up the entrepreneurial focus of part of the countries having economic Independence. Due to the strong competition process as a result of economic globalization, the formation of clusters and the management of knowledge and the quality systems within companies have the fundamental significance for the survival of the company in a long term. Through this paper, there is an interacting analysis between clusters, knowledge management and the service quality systems, specially those applied to family companies.

\section{RÉSUMÉ}

$\mathrm{L}$ es entreprises familiales constituent le noyau entrepreneurial d'une large partie des pays indépendamment de leur niveau de développement. Etant donné le processus de concurrence exacerbée qui est le fruit de la globalisation économique, la création de pôles de compétences et la gestion de la connaissance et des systèmes de qualité des entreprises relève une importance fondamentale pour la survie à long terme de l'entreprise. Tout au long de ce travail une analyse de l'interaction entre les pôles de compétences, la gestion de la connaissance et les systèmes de qualité des entreprises sera réalisée dans le cadre spécifique des entreprises familiales..
Empresas de familia Clusters

Gestión del conocimiento Calidad

\section{Key Words}

Family Companies Clusters

Knowledge Management

Quality

\section{Mots clefs}

Entreprises familiales

Pôles de compétences

Gestion de la connaissance

Qualité 


\section{Introducción}

a formación de clusters en las economías constituye 乙una de las vías para internalizar los procesos de $\mathrm{I}+\mathrm{D}+\mathrm{i}$ en las empresas, así como para modernizar los tejidos productivos y comerciales de los países. De acuerdo con Prahalad y Hamel (1990) las capacidades financieras, comerciales y humanas de las empresas constituyen la base de unas competencias que, suficientemente ampliadas, pueden hacer que las corporaciones tengan un desarrollo sostenible en el tiempo.

Sin embargo, en el mundo actual caracterizado por competir en la globalización no basta con la formación de clusters para tener éxito empresarial, sino que la gestión del conocimiento adquiere una importancia fundamental tanto para conseguir posiciones de liderazgo sostenibles en el tiempo como para satisfacer a los stakeholders. De esta realidad no están exentas las PyME familiares, quienes están obligadas a optimizar el uso de unos recursos que suelen ser más escasos que los que están a disposición en las empresas transnacionales y multinacionales.

A lo largo de este trabajo se analiza la relación entre los clusters y la gestión del conocimiento como instrumentos de creación de riqueza y bienestar económico y social, aplicándose esta relación a las empresas familiares. Para ello comenzaremos estudiando cómo la gestión del conocimiento y los clusters son generadores de ventajas competitivas. A continuación se analizará cómo la calidad influye en las corporaciones y se darán unas pautas para su implantación. Finalizaremos con unas conclusiones.

\section{La gestión del conocimiento como ventaja competitiva}

$\mathbf{E}^{\mathrm{n}}$ los últimos años ha adquirido un papel relevante en as empresas la rapidez en la transmisión de la información fruto del avance de la tecnología. Las empresas están sometidas a grandes cambios, fruto de las Nuevas Tecnologías de Información y del Conocimiento (NTIC), lo que influye en su política y estrategia corporativa al haber cambiado de un paradigma tayloriano de solo producción industrial, a otro basado en el conocimiento y la adquisición de competencias (Ramachandran y Sougata, 1996). El cambio tecnológico está afectando aceleradamente al entorno empresarial actual fruto de la globalización económica, lo que está favoreciendo la formación de alianzas estratégicas entre las empresas ${ }^{1}$. Como resultado, los recursos productivos compiten a escala mundial principalmente a partir de ventajas porterianas de primer orden o de rango superior (Saiz, 2004).
De esta realidad empresarial no está exenta la Unión Europea (UE). En su Informe Anual sobre la Economía Digital para 2007, las Tecnologías de la Información y la Comunicación

(TIC) generan el 50\% del crecimiento de la UE (Comisión Europea, 2007). El objetivo principal de las empresas es conseguir un equilibrio dentro de estas tendencias que le permita mantenerse en el mercado, utilizando el desarrollo tecnológico como valor estratégico dentro del negocio de la misma.

\footnotetext{
1 Una buena aproximación a las alianzas empresariales como instrumento de ganancias de competitividad se encuentra en García-Ochoa, M. (2008).
} 
La utilización de la tecnología permite a las organizaciones conseguir ventajas competitivas de primer orden (Porter, 1990) o de rango superior que fortalece a la empresa en su conjunto y la diferencia de la competencia. Esto permite a la corporación ofrecer productos y servicios a nichos de mercado diferenciados en un ambiente marcado por la competitividad y la innovación constante.

Pero el presente y el futuro van más allá de la mera recopilación de la información y el conocimiento. El objetivo final de esta Era de la Información o Cibersociedad

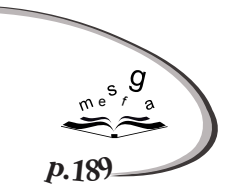

Tecnológica Postindustrial (CTP) (Saiz, 2006), reside en la adecuada gestión de esa información y conocimiento, de tal forma que nos permita obtener un plus o un valor añadido sobre el mero hecho de conocer o disponer de esa información, aprovechando las ventajas de la interacción de todo el volumen de información.

\subsection{La información como un bien intangible}

La obtención de una información transparente por parte de los stakeholders de una organización es fundamental para que la misma logre unas elevadas tasas de crecimiento sostenible en el tiempo. La razón de este hecho viene dado por la importancia que tienen, tanto clientes como stakeholders (principalmente accionistas), en la generación de flujos de caja (cash flow) presentes y futuros, para lograr así la supervivencia de la organización.

La naturaleza y las características de la información determinan las reacciones de los agentes económicos, Así, cuando la información es tangible, objetiva y transparente la reacción de los stakeholders, principalmente inversores, es pequeña, no siendo así cuando hay opacidad en la misma (Daniel y Titman, 2001), produciéndose incluso situaciones de pánico financiero. De ahí que sea fundamental que existan unos canales de información veraces y transparentes para dar estabilidad a los mercados y, por extensión a las empresas.

La gestión de la información y el conocimiento es un recurso de las empresas de hoy en día que, dadas sus características peculiares como es el caso de la intangibilidad, no se contempla frecuentemente como un recurso propiamente dicho dentro de la empresa, aunque existen esfuerzos para valorarla, tanto en estudios generales (Fraser, Tarbert y Hong Tee, 2009; Barnes y McClure, 2009), como en industrias específicas tanto sectorial como geográficamente (Gu y Wang, 2005; De y Dutta, 2007; O’Mahony y Vecchi, 2002; Van Ark et al, 2009; Bontempi y Mairesse, 2008).

En general, la información tiene una serie de características muy peculiares, según Aja (2002), expandible, comprimible, sustituible, difusa y compartida. Fruto de esta intangibilidad, en ocasiones no se le da la relevancia que tiene como recurso en la empresa, llegando a carecer en ocasiones de valor dentro de la misma y de la atención adecuada. La información, desde esta perspectiva, erróneamente no es considerada como un activo de la organización, lo que origina ineficiencias X en las organizaciones.

Sin embargo, el valor asociado al recurso de la información y el conocimiento es incalculable en una sociedad de la información. Tanto es así que se hace necesario para las empresas disponer de un sistema de gestión del conocimiento y de la información adecuado a las necesidades y características tanto de la empresa, como de la información manejada por la misma.

La importancia de la gestión del conocimiento radica en ser la llave que permitirá a una empresa genérica pasar de la mera generación de datos (Martínez, 1999) a la generación de información, y de esta al conocimiento y la sabiduría dentro de la organización.

En ciertas ocasiones se ha referido a estos intangibles como activos relacionales en la empresa hablando en un entorno económico (Boisier, 2001; Storper,1997), asegurando la innovación y competitividad. Al ser relacionales permiten la generación de beneficios a partir de trabajo en equipo multidisciplinares, tanto en entornos nacionales como internacionales. Trabajo en equipo en el que asumir los roles entre los distintos miembros del equipo es fundamental, así como la facilidad y rapidez en la puesta en marcha
"La importancia de la gestión del conocimiento radica en ser la llave que permitirá a una empresa genérica pasar de la mera generación de datos a la generación de información, y de esta al conocimiento y la sabiduría dentro de la organización". (Martínez, 1999) 
de mecanismos de transmisión de información veraz y transparente entre los distintos miembros del equipo para lograr unos altos niveles de productividad y eficiencia en la organización. Pero no sólo es suficiente con esto, sino que las organizaciones que necesitan, adquieren como propio y valoran este recurso, muestran una serie de características:

\Presentan una fuerte capacidad de cohesión y sentimiento generalizado de identidad común (Aja, 2002).

\ Tienen una tendencia innata hacia la internacionalización, ya sea para ampliar los nichos de mercado existentes, como para encontrarnuevos yacimientos de empleoy posibilidades de nuevos negocios.

¿ Disponen de una alta capacidad de aprendizaje y adaptación a los cambios (Aja, 2002).

\ Tienen la tendencia, de forma innata en su filosofía corporativa, a satisfacer a los stakeholders de la organización, principalmente a los accionistas, lo que la lleva a procesos de crecimiento sostenible en el tiempo.

〕 Dan una importancia creciente a la formación especializada dentro de la organización, incluyéndolo como requisito imprescindible para promocionar dentro de la misma.

$\downarrow$ Tienen una fuerte tendencia a formar pliopolios negativos, generados a partir de procesos de fusiones y absorciones de empresas, lo que lleva a fortalecer su posición competitiva en el mercado.

¿ Son tolerantes con el pensamiento y la experiencia poco convencional (Aja, 2002).

$\downarrow$ Presentan un alto grado de confianza y apoyo entre los recursos humanos de la empresa (Pavez, 2000).

『 Tienen un fuerte afán de superación y están orientadas hacia la excelencia.

Son estas características y en especial la última de ellas las que permiten explotar las ventajas que ofrece la adecuada gestión de la información y el conocimiento convirtiéndolas en una sustancial ventaja competitiva para la empresa.

\subsection{La organización inteligente como paradigma empresarial}

Se define como organización inteligente a la capacidad de la empresa, con independencia de su tamaño, características y posición competitiva en su sector, para procesar conocimiento para conseguir soluciones óptimas que permitan a la misma lograr su supervivencia en entornos competitivos. Podemos hablar así del concepto de organización inteligente en el mercado actual, donde su mayor potencial radica en la capacidad de aprender (Pavez, 2000), extendiendo el concepto a todo el contexto organizacional, como un producto de inteligencia distribuida. Dicha capacidad de aprender permite a la organización individual como en clúster, hacer frente a nuevas necesidades de los clientes nuevos como existentes, y así lograr procesos de crecimiento sostenible en el tiempo. De ahí que el aprendizaje sea incluso vital no sólo para el mantenimiento de la empresa en posiciones de liderazgo competitivo, sino para la mera supervivencia de la misma en entornos económicamente hostiles y competitivos.

La característica distintiva de las organizaciones inteligentes viene dada por su capacidad de adaptación en entornos cambiantes gracias a una nueva estructura cognitiva formada

por el capital

intelectual de la

organización

(Bratinau, Vasilache

y Jianu, 2006). Dicha

capacidad cognitiva

se genera, sobre

todo, en clusters

de empresas que

compiten en entornos

globales, al ser funda-

mental para el logro

de éxito empresarial a

escala mundial una

"La característica distintiva de las organizaciones inteligentes viene dada por su capacidad de adaptación en entornos cambiantes gracias a una nueva estructura cognitiva formada por el capital intelectual de la organización (Bratinau, Vasilache y Jianu, 2006)".

así como una rápida toma de decisiones.

Una de las ventajas competitivas más relevantes para la empresa es la de preparar el escenario adecuado para la correcta, ágil y fundamentada toma de decisiones a nivel estratégico. Esto aportará la ventaja competitiva en la flexibilidad como en la adaptación a los cambios y 
necesidades del mercado en cada momento. Esta ventaja reforzará la estrategia de empresa garantizando así su permanencia y fortalecimiento en el mercado.

Resultado de todo ello, las ventajas que puede aportar el proceso de aprendizaje a la gestión del conocimiento dentro de la empresa se pueden sintetizar en los siguientes campos:

$\checkmark$ Permite la apertura de nuevos negocios, lo que aumenta la competitividad y la eficiencia de la empresa, asegura la presencia de la compañía en el mercado y aumenta el liderazgo de la empresa en el sector.

Eleva el rendimiento, la eficacia de la empresa, el ciclo de desarrollo de los productos, y como resultado el EBITDA (Earnings before Interests, Taxes, Depreciation and Amortization).

\ejora la satisfacción de usuarios y clientes, al gestionar adecuadamente las relaciones con clientes, usuarios y proveedores.

\Aumenta la comunicación, conocimiento y capacitación de los empleados de la empresa, y así ofrecer a los clientes una imagen óptima de empresa. Proporciona, en definitiva, espacios de trabajo, compartiendo las buenas prácticas, lo que permitirá gestionar la propiedad intelectual.
En un entorno competitivo la estructura de la empresa ha de ser orgánica, es decir, adaptable al medio al igual que lo hacen los seres vivos (Mintzberg, 1997). Como resultado, la empresa aprende más rápido y se adapta a las siguientes cinco fases consecutivas (Choo y Bontis, 2002): inicio y orientación preliminar; diagnóstico y determinación de objetivos; realización de nuevos objetivos; evaluación, y seguimiento. Durante la fase de evaluación los directivos de la empresa analizan si la organización está aprendiendo, mientras que en la última fase el aprendizaje obtenido se aplicará para resolver futuras situaciones.

Por otra parte, cuando una empresa es orgánica se caracteriza por su dinamismo y por su capacidad para diseñar estrategias corporativas que logren éxito en entornos competitivos. Al contrario de quienes opinan que lo más importante de la empresa es el cliente, en las organizaciones inteligentes actuales lo primordial es tener un capital intelectual altamente motivado, ya sea con una remuneración económica y en especie al menos similar a la de la competencia, o mediante una carrera profesional justa y basada en la meritocracia. Las empresas que no tengan, de forma ideal, estas dos políticas para motivar a sus empleados, irán perdiendo los mismos a medida que vayan siendo captados por la competencia. Hoy en día, por tanto, es más valioso el capital intelectual que trabaja en la organización que los propios clientes.

\section{Los clusters de empresas familiares: claves para su formación}

D e esta situación de competencia para captar al mejor capital intelectual no está exenta la empresa familiar. Siguiendo a Shanker y Astrachan (1996) se concibe a la empresa familiar desde una triple clasificación: una definición amplia de la empresa familiar en la que la familia únicamente mantiene el control en la dirección estratégica de la misma sin que entre en la gestión, por lo que controla en la sombra a la corporación; una definición intermedia de la empresa familiar en la que, además de lo anterior, la familia coopera con el gestor en la resolución de los problemas, por lo que se involucra más en los resultados finales de la misma; y una definición estrecha de la empresa familiar en la que coinciden gestión y propiedad, por lo que es la familia quien lleva las riendas de su negocio.

Basándose en una escala Gutmann y aplicándola a 885 pequeñas y medianas empresas (PyME) holandesas, Uhlaner (2002) desarrolla un índice para medir la existencia de empresas familiares. A efectos operativos la escala Gutmann se puede aplicar tanto en análisis cuantitativo, principalmente análisis factorial y tabulación cruzada, como en el cualitativo, siendo en nuestra opinión más sencilla y factible para la PyME familiar la aplicación de la escala Gutmann en el análisis cualitativo. 
El procedimiento se caracteriza por realizarse en tres fases: preparación de un cuestionario con preguntas cerradas de elección múltiple o basadas en una escala Lickert (preferiblemente de 1 a 5 como, opcionalmente, de 1 a 7) para ser respondidas únicamente por los empresarios de las PyME; clasificación de las preguntas en dos tipos en función de la dificultad de su aplicación en la empresa (fácil aplicación y difícil aplicación), y tabulación y análisis de las respuestas sin que sea necesaria una segunda ronda. Aquellas PyME que haya contestado positivamente en un número prefijado de estrategias de difícil aplicación en la empresa van primeras en el índice, al mostrar una mayor disposición para aprender, un mayor impulso para asumir riesgos y, al menos a priori, una mayor rentabilidad a partir del binomio riesgorentabilidad.

A medida que la empresa familiar va creciendo, sobre todo si pasa de una generación a otra, una vez resueltos los problemas de la sucesión, se puede ir convirtiendo en un clúster, al representar los clusters familiares a concentraciones de empresas que son capaces de ser más competitivas en función de sus relaciones (Feser, 1998), generar sinergias por su proximidad geográfica e interdependencia, incluso aunque su escala de empleo no sea importante y por generar valor (Roelandt y Den Hertog, 1999).

En la empresa familiar desaparece la tradicional diferencia entre clúster y distrito industrial, porque mientras en un clúster se produce un creciente nivel de aglomeración, en el distrito industrial se pone énfasis en los valores y normas comunes de las empresas del grupo que, al ser familiar, suelen ser más parecidas que en clusters no familiares. Por ello, los clusters familiares son empresas próximas con fuertes relaciones verticales y horizontales que incluyen una infraestructura de apoyo localizada y una visión compartida del crecimiento del negocio basada en la cooperación (Cooke y Huggins, 2002). Hoy en día la estructura empresarial de las economías del mundo, con independencia de su estado de desarrollo, pasa por estar basada en empresas familiares. Tipo de empresas que se unen y se concentran geográficamente al buscar proveedores especializados, empresas de industrias relacionadas e instituciones asociadas en un campo determinado que compiten y cooperan a la vez (Porter y Stern, 1998).

Fruto de su complejidad y dispersión, existe una gran diversidad de clusters $^{2}$. En lo que respecta a los clusters de empresas familiares son fundamentalmente distritos industriales canónicos caracterizados por una multiplicidad de PyME familiares con fuertes relaciones entre sí y un sentimiento de pertenencia a la comunidad en donde están establecidas. De ahí que, y sobre todo en poblaciones pequeñas y medias, se produce una identificación del clúster con el producto ofrecido al mercado, tal y como sucede, por ejemplo en las empresas familiares, todas ellas en segunda o tercera generación. Este hecho se da en industria transformadora de productos básicos alimenticios, tal y como se muestra en el cuadro 1.

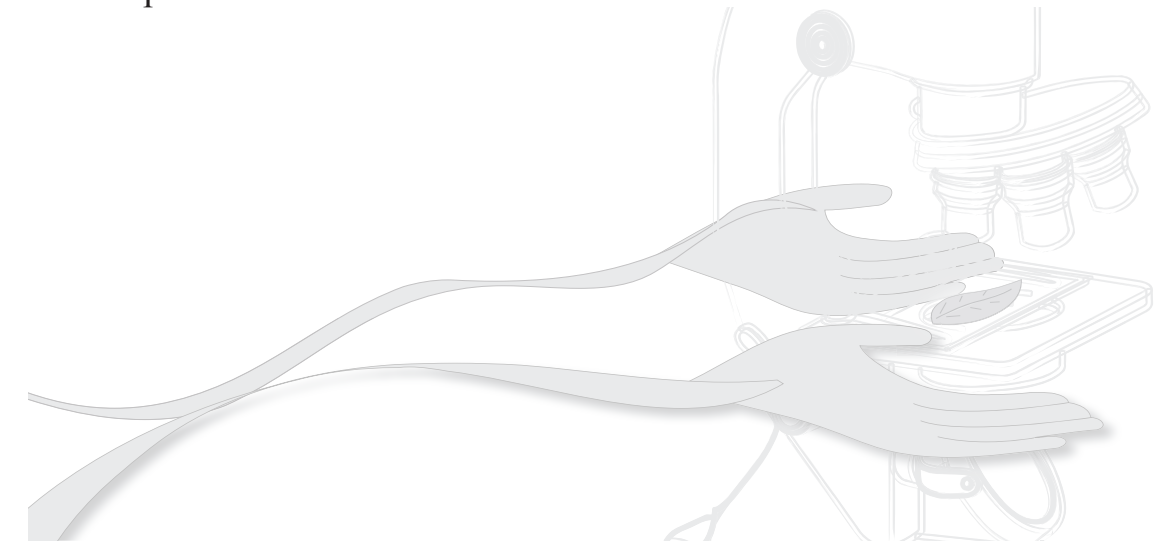

\footnotetext{
2 Paniccia (2006) distingue entre: (1) Distritos industriales canónicos; (2) Distritos industriales diversificados o urbanos; (3) Plataformas satélites o aglomeraciones hub and spoke; (4) Áreas co-localizadas; (5) Aglomeraciones o distritos industriales concentrados o integrados; y (6) Aglomeraciones basadas en I+D+i.
} 


\begin{tabular}{|c|c|c|}
\hline Producto & Localidad & Provincia \\
\hline Aceite de oliva & Andújar & Jaén \\
\hline Ajo & Chichón & Madrid \\
\hline Anchoas & Santoña & Santander \\
\hline Cava & San Sandurní de Anoia & Barcelona \\
\hline Chorizo & Cantimpalos & Segovia \\
\hline Fresón & Lepe & Huelva \\
\hline Jamón serrano & Jabugo & Huelva \\
\hline Morcilla de arroz & Villarcayo & Burgos \\
\hline Pimientos & Padrón & Pontevedra \\
\hline Polvorones & Estepa & Sevilla \\
\hline Turrón (todas las variedades) & Jijona & Alicante \\
\hline Vino (uva Mencía) & Almázcara-Cacabelos & El Bierzo (León) \\
\hline
\end{tabular}

Cuadro 1.

Identificación del producto con clusters familiares locales (sólo España).

Fuente: elaboración propia

Los clusters de empresas tienen un fuerte impacto regional (Porter, 2003), sobre todo si se encuentran en los primeros estadios de desarrollo en donde es mayoritaria el número de clusters familiares. Como resultado, se produce una fuerte movilidad laboral, principalmente horizontal, lo que acelera de forma indirecta la transferencia de $\mathrm{I}+\mathrm{D}+\mathrm{i}$ entre las empresas del clúster. Esta transferencia tecnológica y de procesos es aún mayor a medida que los recursos humanos sean más escasos y estén más especializados.

Una de las claves para que los clusters de empresas familiares se puedan formar, viene dada por la confianza entre las partes en la que la inteligencia emocional (Goleman,1995) juega un papel importante. Dicha confianza aparece en las organizaciones inteligentes quienes saben valorar correctamente e incentivar a su capital intelectual para que dé lo mejor de sí en un entorno competitivo y ofrezca su conocimiento tácito para el beneficio de la organización (Roos et al, 1997).

Otro factor que incentiva la formación de clusters es la facilidad para la movilidad laboral, tanto horizontal como vertical, sobre todo para los recursos humanos más especializados. Esto hace que se produzca una concentración de empresas por sectores o por productos, por lo que dicha aglomeración obedece tanto a factores económicos (economías de urbanización y establecimiento) como a factores productivos (transferencia de las 7-K Know-how, Know-who, Know-whom, Know-what, Know-why, Knowwhen y Know-whose; Saiz, 2010) entre las empresas del clúster, movilidad laboral, aceleración en la transferencia de $\mathrm{I}+\mathrm{D}+\mathrm{i}$, fortalecimiento en las relaciones entre los miembros del clúster,...).

El alcance de un buen nivel educativo en la población favorece la creación de clusters a nivel regional como local, al haber una relación directa entre un mayor nivel educativo y la generación de innovaciones en las empresas, como sucede en los países con elevados niveles de capital-riesgo. Todo ello genera ventajas competitivas sustentadas por los bienes intangibles de la empresa, en especial la reputación corporativa (Verbeke y Frances, 2006), lo que genera, además, goodwill para la empresa.

Junto a la iniciativa privada, y fruto de un modelo económico ordoliberal generador de efectos crowdingin para la empresa privada, una política activa de ayudas públicas $\mathrm{y}$, de forma simultánea, de reducciones de impuestos favorecen la atracción de clusters a las regiones fiscalmente más atractivas. Esta situación es especialmente palpable en aquellos países en donde las regiones tienen regímenes fiscales distintos, tal y como sucede en España. Fiscalidad más favorable que acelera los procesos de $\mathrm{I}+\mathrm{D}+\mathrm{i}$ en la región, siempre que exista $e x$ ante una buena red de infraestructuras, principalmente de transporte y telecomunicaciones.

Por otro lado, el tamaño de mercado potencial es un factor que influye en la formación de clusters. En este sentido, las barreras técnicas y las imposiciones lingüísticas por factores políticos debilitan la formación de clusters 
al crear mercados cautivos de tamaño reducido, como sucede en España en los casos catalán y vasco. Un mercado cautivo, al hacerse estrecho a medio plazo, provoca la necesidad de estar financiado por los poderes públicos para que sea sostenible, lo que es injusto desde un punto de vista económico. Por eso, los mercados estrechos diferenciados por valores culturales y lingüísticos propios de una minoría, están llamados a languidecer primero para posteriormente desaparecer al no poder competir sin ayudas públicas, al ser ineficientes, en entornos globalizados.

Otro factor clave para la formación de clusters viene dado por la existencia de bajos niveles de exposición al riesgo. Gran parte de las PyME, si desean
"El alcance de un buen nivel educativo en la población favorece la creación de clusteres a nivel regional como local, al haber una relación directa entre un mayor nivel educativo y la generación de innovaciones en las empresas, como sucede en los países con elevados niveles de capital-riesgo". sobrevivir en un entorno competitivo, han de calibrar bien su exposición al riesgo. Es por ello que siempre son bienvenidas las incubadoras de empresas para que las PyME puedan disminuir su grado de exposición al riesgo. Estas tienen cada vez más relación con entornos universitarios, al constituir una vía de desarrollo del espíritu emprendedor.

La disposición de unos fuertes niveles de solvencia comercial y financiera constituye una garantía de éxito empresarial a medio plazo siempre que se mantengan en el tiempo. En este sentido,

la política estratégica de la corporación es fundamental para lograr que la empresa crezca y se desarrolle en un entorno competitivo para mantenerse así a largo plazo.

\section{Cuadro 2}

Diferentes enfoques en la identificación y medición de clusters

\begin{tabular}{|l|c|c|c|c|}
\multicolumn{1}{|c}{$\begin{array}{c}\text { Concepto de } \\
\text { clúster }\end{array}$} & $\begin{array}{c}\text { Profundidad } \\
\text { conceptual }\end{array}$ & $\begin{array}{c}\text { Metodología } \\
\text { empírica }\end{array}$ & $\begin{array}{c}\text { Facilidad de } \\
\text { medición }\end{array}$ & $\begin{array}{c}\text { Soporłe } \\
\text { empírico }\end{array}$ \\
\hline $\begin{array}{l}\text { Co-localización } \\
\text { po-localización y }\end{array}$ & Superficial & Top-down & $\begin{array}{c}\text { Fácil de medir } \\
\text { (Cuantitativo) }\end{array}$ & $\begin{array}{c}\text { Evidencia } \\
\text { indirecta }\end{array}$ \\
\hline $\begin{array}{l}\text { Tablas input-output y } \\
\text { complementariedades }\end{array}$ & $\downarrow$ & $\downarrow$ & $\downarrow$ & $\downarrow$ \\
\hline $\begin{array}{l}\text { Co-localización y des- } \\
\text { empeño superior }\end{array}$ & $\downarrow$ & $\downarrow$ & $\downarrow$ & $\downarrow$ \\
\hline $\begin{array}{l}\text { Externalidades Marsha- } \\
\text { llianas }\end{array}$ & $\downarrow$ & $\downarrow$ & $\downarrow$ & $\downarrow$ \\
\hline Redes de empresas & $\downarrow$ & $\downarrow$ & $\downarrow$ & $\downarrow$ \\
\hline Colaboración explícita & $\downarrow$ & $\downarrow$ & $\downarrow$ & $\downarrow$ \\
\hline $\begin{array}{l}\text { Diseminación artificial } \\
\text { de conocimiento }\end{array}$ & Profunda & Bottom-up & $\begin{array}{l}\text { Difícil de } \\
\text { medir (cualitativo) }\end{array}$ & $\begin{array}{c}\text { Evidencia } \\
\text { directa }\end{array}$ \\
\hline
\end{tabular}

Fuente: Blázquez (2008) a partir de Martin y Sunley (2003) y Swann (2002).
Otro de los factores más destacables para tener éxito en la formación de clusters familiares viene dado por la firma de protocolos familiares que garanticen la ausencia de problemas y tensiones durante la sucesión de la corporación. Sólo un 3,5 por ciento de las empresas familiares llegan a la cuarta generación (Saiz, 2009).
Por último, uno de los factores que está teniendo cada vez más importancia en las economías más desarrolladas del planeta caracterizadas, entre otros aspectos, por tener clientes altamente exigentes, viene dado por la adopción de sistema de calidad en las empresas. Sistemas cuya gestión es fundamental para tener éxito, tal y como veremos en el epígrafe siguiente. 


\section{El sistema de calidad en la empresa familiar}

U sistema de gestión de la calidad dentro de una organización es una de las piezas claves para el funcionamiento de la misma y para su supervivencia en un futuro más o menos inmediato. Igualmente, es importante la integración de dicho sistema de calidad con otros sistemas dentro de la empresa, como puede ser el caso del Sistema de Gestión Medioambiental o el Sistema de Prevención de Riesgos Laborales.

Es fundamental para optimizar el funcionamiento de la empresa que todos los sistemas que forman parte de la misma se encuentren integrados y en armonía. De esta forma, le permitirán obtener el mayor número de ventajas fruto de la combinación de todos sus sistemas, y reducir los posibles riesgos asociados a un conjunto de sistemas erróneamente integrados o con deficiencias en la integración global.

Todos los sistemas deben estar alineados con la política y la estrategia de la empresa, y ser fieles a los objetivos marcados por ella, de esta forma facilita su alcance y cumplimiento. Las ventajas y aportes de un sistema de calidad en la empresa, pueden ser las que se citan a continuación:

$\checkmark$ Enfocar la cultura empresarial a los requisitos del cliente, realizando una mejora continua en los procesos (en caso de que sea necesario reajustarlos tras el paso del tiempo), productos y servicios para asegurar su satisfacción con los mismos y conseguir la aceptación total por parte de los clientes.

Lograr que la empresa sea más competitiva, al reducir costos en los procesos, aumentando la productividad, efectividad y utilidad de la empresa, y certificando si procede su competitividad internacional.

$\checkmark$ Tener criterios de medición e indicadores y poderlos comparar con las mejores prácticas para conocer fortalezas y debilidades de la empresa y establecer las estrategias necesarias de mejoramiento.

\ejorar la calidad de vida del personal de la empresa, cambiando la actitud del mismo.

\subsection{Requisitos e implantación de un sistema de gestión del conocimiento en la empresa familiar}

Los sistemas de la información y el conocimiento surgen del continuo avance que estamos experimentando en las Tecnologías de la Información y el Conocimiento (TIC) desde el punto de vista de la globalización (Boisier, 2001).

Los objetivos que se plantean en las organizaciones con la implantación de un sistema de gestión del conocimiento pueden resumirse en los siguientes puntos:

\úsqueda de un enfoque estratégico hacia la mejora continua y hacia la excelencia.

¿ Relacionar la estrategia de empresa con la aplicación de la gestión de la información y del conocimiento (Pavez, 2000), y perseguir que la estrategia de la Gestión del Conocimiento siga los principios de la estrategia de empresa.

¿ Búsqueda de eliminación de los defectos en los productos o servicios ofrecidos aprovechando el conocimiento y la experiencia adquiridos.

〕 Disminuir los tiempos de producción y de mejora de los productos o servicios ya existentes.

En consecuencia de los dos puntos anteriores, también se buscará reducir los costos asociados a la no calidad de los productos o servicios.

\ Impulsar la innovación e investigación, facilitando la aportación personal y las mejores prácticas, y en consecuencia, la competitividad de la empresa.

\ Búsqueda de un espíritu de unidad e integración entre los trabajadores de la empresa, propiciando así un adecuado ambiente de trabajo en colaboración y puesta en común del conocimiento. 
\ Apoyo incondicional de la dirección de la empresa durante la implantación de la gestión del conocimiento.

No tiene sentido en ningún caso realizar una implantación de un sistema general de calidad de forma brusca, sino que es conveniente esperar la mejor oportunidad de arrancar el proyecto, y someter a contraste los resultados obtenidos a lo largo del mismo con los resultados inicialmente previstos, para estar en disposición de controlar la evolución de dicho proyecto.

Un modelo de gestión de la información y del conocimiento debe contemplar una serie de procesos a tener en cuenta en su implantación en la empresa. Estos son:

\ Adquisición de la información y del conocimiento.

\ Diseño de los mapas del conocimiento adecuados a la situación de la empresa, individuales o colectivos, con el conocimiento, capacidad y competencia actuales y esperadas.

¿lección o diseño de las herramientas adecuadas para la gestión del conocimiento. Podemos tener en cuenta diferentes tipos de herramientas (Núñez y Núñez, 2005):

- Herramientas de búsqueda y recuperación de la información: motores de búsqueda.

- Herramientas de filtrado y personalización de la información.

- Tecnologías de almacenamiento y organización de la información, entre los que hay que citar, entre otros, el Sistema de Gestión de Base de datos (SGBD) y el Data Warehouse.

- Herramientas de análisis de información, entre los que destacan el Data Mining y las redes neuronales.

- Sistemas de gestión de flujos y comunicación, como el Diagrama de Flujo de Datos (DFD), los portales corporativos y el workflow.
- Herramientas de aprendizaje y comercio electrónico (sistemas de e-Learning y e-Commerce).

- Sistemas de gestión empresarial, como el Enterprise Resource Planning (ERP) y el Customer Relationship Management (CRM) (Saiz y De la Antonia, 2009).

$\checkmark$ Refinamiento y adaptación de la información y conocimiento recopilados al modelo.

A Almacenamiento de la información y disposición de recuperación de datos para su uso en caso necesario, a través de las herramientas disponibles o implantadas a tal efecto.

\ Distribución de la información a las partes interesadas.

$\checkmark$ Presentación de los datos al usuario de los mismos con el formato adecuado.

Una barrera para el desarrollo de la gestión de la información y del conocimiento dentro de las empresas reside en la dificultad de selección de un modelo de gestión del conocimiento adecuado a la situación, características y necesidades de la empresa. En muchos casos hay verdadero temor ante la posibilidad de no elegir un modelo adecuado, lo que limita su impulso dentro de la empresa. Por otra parte, tampoco existe un modelo mágico que tenga asegurado el éxito en la implantación dentro de una empresa, ni una serie de reglas infalibles para su elección en función del sector o características de la empresa.

Existen varios modelos de gestión del conocimiento (Lahaba y León, 2001) (Rodríguez, 2006), con sus características y peculiaridades:

Gopal y Gagnon (1995).

¿ La organización creadora de conocimiento (Nonaka y Takeuchi, 1999).

¿ The 10-Step Road Map (Tiwana, 2002).

\ Modelo de gestión del conocimiento desde una visión humanista (R. de Tena, 2004, en Gallego y Ongallo, 2004). 
\ Modelo de implantación de gestión del conocimiento desde la cultura organizacional (Marsal y Molina, 2002).

A pesar de las singularidades de cada uno de los modelos citados como ejemplo, y en general para la multitud existentes, se podrían englobar en estas tipologías según la metodología y objetivos de los mismos:

$\checkmark$ Modelos de almacenamiento, acceso y transferencia (Rodríguez, 2006). Consideran la información y el conocimiento como independientes de las personas que lo generan y utilizan. Están centrados en el almacenamiento de conocimiento propiamente dicho, y en la existencia de repositorios de conocimiento que facilitarán la búsqueda posterior. En ocasiones esta tipología podría contemplarse desde un punto de vista más tecnológico, haciendo uso más extenso de sistemas y herramientas tecnológicas adecuadas para la creación y el almacenamiento, pero sin perder de vista este enfoque del almacenamiento propiamente dicho, pero incluyendo por ejemplo motores de búsqueda, datawarehouse, entre otros.

〕 Modelos socioculturales (Rodríguez, 2006). Están más enfocados a la creación de una cultura organizacional acorde y basada en la gestión del conocimiento, y en la generación de procesos que permitan la adecuada gestión y divulgación del conocimiento dentro de la empresa. Para el desarrollo de esta tipología de modelos hay que contar con la concienciación de todos los empleados en la cultura definida, y con el apoyo incondicional de la dirección de la organización.

Un punto decisivo durante la implantación del modelo de gestión del conocimiento es el diseño de la arquitectura del conocimiento (Pavez, 2000) que establecerá la base lógica y técnica del sistema de información y del conocimiento. y también la adecuada y óptima elección de las herramientas de gestión del conocimiento (Núñez y Núñez, 2005). en estos casos, el propio desconocimiento aleja de la gestión del conocimiento el concepto de recurso dentro de la empresa.

\subsection{Riesgos y dificultades de la implantación del sistema de gestión del conocimiento en la empresa familiar}

Como primer reto o primera barrera a este respecto dentro de la empresa, esta puede encontrarse con la dificultad de llegar a considerar el conocimiento como un activo dentro de la empresa (Lahaba y León, 2001), y reconocer que este se adquiere, se crea, se aplica, se protege y se difunde dentro de la organización. Además de la barrera comentada anteriormente a la implantación de la gestión de conocimiento dentro de la empresa por el desconocimiento del tema por parte de la empresa, nos podemos encontrar con una serie de dificultades, como es el caso de:

¿ El modelo de relación entre las actividades, la competencia y el conocimiento y el análisis de las brechas del conocimiento (Pavez, 2000).

¿ La creación del clima organizacional (Pavez, 2000) para la correcta implantación del modelo de gestión de la información y el conocimiento. En este punto hay que destacar la importancia que tiene la comunicación interna de la organización (Núñez y Núñez, 2005), y que puede planificarse de forma adecuada como base de la gestión del conocimiento.

\La actualización constante de información relativa a clientes, proveedores, colaboradores, reguladores, competidores, etc., a través de las herramientas disponibles para ello en la empresa.

¿ La flexibilización de las estructuras verticales (Núñez y Núñez, 2005) de la organización en estructuras más distribuidas.

¿ La revisión de la seguridad y conexión de toda la organización, que protege esa información poniéndola a disposición de las partes interesadas de forma segura.

Dada la magnitud e importancia de la gestión del conocimiento y de la información en la empresa, la aspiración y tendencia general de las organizaciones en ese afán de gestión adecuada de la información y el conocimiento podría dar en llamarse Business Networking (Österle, Fleisch y Alt, 2000) o interconexión de las empresas como la culminación del proceso de gestión del

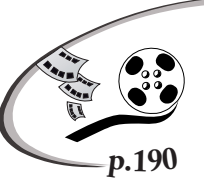
conocimiento. 


\subsection{La adaptación del modelo de gestión del conocimiento al sistema de calidad}

Sin perder de vista estos riesgos y dificultades que una empresa se puede encontrar al implantar un sistema de gestión del conocimiento en la misma, la organización deberá enfocar dicha implantación dentro de las particularidades del sistema de calidad existente o por implantar en la empresa, de tal forma que debe ajustarse al mismo teniendo en cuenta, los siguientes aspectos adicionales:

$\checkmark$ Introducir la cultura de la calidad en la organización, enfocada hacia la atención del usuario, y que convierta la mejora en un proceso dotado de continuidad. En este aspecto, la gestión del conocimiento deberá hacer de facilitador en la divulgación de la política de calidad de la organización entre las partes de la misma, así como la documentación y principios en que se sustenta la calidad de la empresa: manual de calidad, plan de calidad, acuerdos de nivel de servicio o requisitos de producto, registros de calidad, auditorías y planes de acción, entre otros.

Inculcar en general la premisa de hacerlo bien, a la primera vez y siempre, creando constancia y perseverancia a la hora de mejorar los productos o servicios. Hacer propuestas de innovación. El modelo de gestión del conocimiento debe tener su parte de colaboración en este aspecto, recogiendo las mejores prácticas y facilitando su disponibilidad y divulgación entre el personal de la empresa.

〕 Diseñar un programa para el diseño e implantación de los procesos y sistemas que integran el modelo de calidad. El sistema de gestión del conocimiento formará parte del citado programa, con sus procesos asociados y teniendo en cuenta la plataforma tecnológica y el software que requiere.

$\checkmark$ Promover los valores de calidad y comprometerse con el bienestar de la sociedad y la conservación del medio ambiente. El sistema de gestión del conocimiento debe tener presente la importancia de aglutinar la información, buenas prácticas y conocimiento relativos a los proveedores, clientes, usuarios, colaboradores, etc.

A estos aspectos generales se añadirán todos aquellos más específicos del sistema de calidad existente o por implantar en la empresa, o por la combinación de varios de estos modelos coexistiendo en la empresa, como también se dan casos, siempre dependiendo del sector y de la actividad de la empresa en cuestión.

\section{Conclusiones}

L os clusters constituyen una fuente de generación de riqueza y de bienestar económico y social a mediano y largo plazo. Dentro de los clusters se destacan los formados por empresas familiares cuya correcta gestión corporativa es fundamental para tener éxito y lograr incluso la supervivencia de la empresa en un entorno globalizado y altamente competitivo. Por ello, se hace necesaria la combinación de una correcta gestión corporativa con la puesta en marcha de sistemas de gestión de calidad, entre los que destaca el EFQM (European Foundation for Quality Management).

$\checkmark$ El conocimiento es un activo para la empresa que hay que internalizar. Sin internalización no es posible alcanzar posiciones de liderazgo dentro del sector en el que se compite. Liderazgo sostenible ex ante para iniciar un proceso de internacionalización empresarial ex post una vez que la corporación haya alcanzado una mínima masa crítica en términos de un mínimo volumen de negocio, baja exposición al riesgo y generación de flujos de caja (cash-flow) y beneficio. Sin la creación de forma sostenible en el tiempo de estas dos variables, la empresa no podrá internacionalizarse y, en el caso de hacerlo, su grado de riesgo comercial y financiero será demasiado elevado por lo que será insostenible en el tiempo.

La creación de clusters empresariales pasa por la formación de emprendedores, muchos de los cuales nacen 
en las propias empresas familiares o adquieren las mismas tras un proceso de sucesión. Como resultado, es importante la firma de protocolos familiares para evitar tensiones en el momento de la sucesión que llevarían incluso a la desaparición de los clusters familiares.

¿ Las economías más avanzadas del mundo se caracterizan por la constante creación de riqueza por parte de sus empresas. Un tejido empresarial económicamente saneado y bien gestionado con sistemas de calidad supervisados por organismos independientes, lleva hacia el éxito empresarial. En este sentido, las universidades y centros de investigación tienen un papel fundamental que cumplir en este proceso generador de riqueza y bienestar, al ser, junto con las empresas, las transmisoras del conocimiento y las 7-K. Conocimiento adecuadamente protegido jurídicamente al constituir un activo para quien lo posee. De ahí que el logro de altos niveles de seguridad jurídica sea vital para atraer no sólo capital extranjero a la economía, sino también para la generación de un tejido empresarial endógeno que se ha de fortalecer desde las incubadoras de empresas e impulsar desde las aceleradoras de empresas. Sólo así se podrán alcanzar situaciones óptimo paretianas en la economía y se podrán alcanzar los objetivos en términos de bienestar social que se han de lograr en la cibersociedad actual (Joyanes, 1997).

\section{Bibliografía}

- Aja, L. (2002): “Gestión de información, gestión del conocimiento y gestión de la calidad en las organizaciones", mimeo, Hospital Militar Central Dr. Carlos J. Finlay, Ciudad de la Habana (Cuba).

- $\quad$ Barnes, P. y McClure, A. (2009): "Investments in Intangible Assets and Australia s Productivity Growth", Staff Working Papers, n. 901, Comisión de Productividad del Gobierno de Australia.

- $\quad$ Blázquez, M.L. (2008): "La influencia de los clusters en la competitividad de empresas y regiones", en Saiz, J.M. y García-Ochoa, M. (Coords.): “Innovación empresarial y globalización económica”, Madrid: FIEC, pp. 107-138.

- $\quad$ Boisier, S. (2001): "Sociedad del conocimiento, conocimiento social y gestión territorial", mimeo, Pontificia Universidad Católica de Chile.

- $\quad$ Bontempi, M.E. y Mairesse, J. (2008): “Intangible Capital and Productivity: An Exploration on a Panel of Italian Manufacturing Firms”, NBER Working Papers, n. 14108, Junio, Cambridge (Ma.): National Bureau of Economic Research.

- Bratinau, C.; Vasilache, S. y Jianu, I. (2006): “In search of intelligent organizations”, Management \& Marketing, vol. 1, n. 4, Bucarest (Rumania): Academia de Estudios Económicos, pp. 71-82.

- Calvo, N.; García, M.T. y Mariz, R. (2003): "Análisis dinámico de las políticas de recursos humanos como creadoras de capital intelectual. El caso de la consultoría en Europa", mimeo, Universidad de La Coruña.

- Colle, R. (2005)[en línea]: "Procesos documentales y gestión del conocimiento", Revista Razón y Palabra, n. 46, Agosto-Septiembre, ITESM Campus Ciudad de México. Disponible en http://www.razonypalabra.org.mx/ anteriores/n46/rcolle.html] Última visita: 5 de abril de 2010.

- Comisión Europea [en línea](2007): “Information Space Innovation \& Investment in RAC”, Inclusion, IP/07/453, 30 de marzo, Bruselas (Bélgica). Disponible en http://ec.europa.eu/information_society/eeurope/i2010/

Última visita: 15 de febrero de 2010. 
- Cooke, P. y Huggins, R. (2002): "High Technology Clustering in Cambridge”, en Amin, S.A.; Goglio, F. y Sforzi, A. (Eds.), The Institutions of Local Development, Londres (Reino Unido): IGU.

- Choo, C.W. y Bontis, N. (Eds.) (2002): “The Strategic Management of Intellectual Capital and Organizational Knowledge”, Oxford (Reino Unido): Oxford University Press.

- $\quad$ Daniel, K.D. y Titman, S. (2001): “Market Reactions to Tangible and Intangible Information“, EFMA 2001 Lugano Meetings, Suiza.

- De, S. y Dutta, D. (2007): "Impact of Intangible Capital on Productivity and Growth: Lessons from the Indian Information Technology Software Industry”, Economic Record, vol. 83, n. s1, Septiembre, The Economic Society of Australia, pp. S73-S86.

- $\quad$ Feser, E. J. (1998): “Old and new theories of industry clusters”, en Steiner, M. (Ed.) Clusters and Regional Specialisation, Londres (Reino Unido): Pion, pp. 18-40.

- $\quad$ Fraser, I.; Tarbert, H. y Hong Tee, K.H. (2009): "Do the financial statements of intangible-intensive companies hold less information content for investors?”, Applied Financial Economics, vol. 19, n. 17, Londres (Reino Unido): Taylor \& Francis, pp. 1433-1438.

- Goleman, D. (1995): “Emotional Intelligence: Why it Can Matter More Than IQ?”, Nueva York (EE.UU.): Bantam Books.

- Gu, F. y Wang, W. (2005): “Intangible Assets, Information Complexity, and Analysts' Earnings Forecasts”, Journal of Business Finance \& Accounting, vol. 32, n. 9-10, Blackwell Publishing, pp. 1673-1702.

- Joyanes, L. (1997): “Cibersociedad. Los retos sociales del siglo XXI”. Madrid (España): McGraw-Hill.

- $\quad$ Lahaba, Y.N. y León, M. (2001): "La gestión del conocimiento: una nueva perspectiva en la gerencia de las organizaciones”. ACIMED, vol. 9, n. 2, Dirección de Postgrado, Universidad de La Habana (Cuba), pp. 121-186.

- Martin, R. y Sunley, P. (2003): “Deconstructing Clusters: Chaotic Concept of Policy Panacea”.Journal of Economic Geography, 3, pp. 5-35.

- Martínez, J. (1999): "El salto desde la gestión de información a la gestión del conocimiento". Scire, Grupo de Tecnologías de la Información, Departamento de Información y Documentación, Universidad de Murcia (España), vol. 5, n. 1, Enero-Junio, pp. 41-54.

- Mintzberg, H. (1997): “The Structuring of Organizations: A Synthesis of the Research”. Nueva York (EE.UU.): Prentice Hall.

- $\quad$ Núñez, C.I. y Núñez, Y. (2005): "Propuesta de clasificación de las herramientas-software para la gestión del conocimiento", ACIMED, vol. 13, n. 2, Dirección de Postgrado, Universidad de La Habana (Cuba), pp. 125-132.

- O'Mahony, M. y Vecchi, M. (2002): “Do Intangible Investments Affect Companies'Productivity Performance?”, NIESR Discussion Papers, 201, Londres (Reino Unido): National Institute of Economic and Social Research.

- Österle, H.; Fleisch, E. y Alt, R. (2000): “Business Networking: Shaping Enterprise Relationships on the Internet”. Berlin-Heidelberg (Alemania): Springer-Verlag.

- Paniccia, I. (2006): "Cutting through the Chaos: Towards a New Typology of Industrial Districts and Clusters". en Asheim, B.; Cooke, P. y Martin, R. (Eds.): Clusters and Regional Development: Critical Reflections and Explorations, Londres (Reino Unido): Routledge. 
- Pavez, A.A. (2000): "Modelo de implantación de gestión del conocimiento y tecnologías de información para la generación de ventajas competitivas". Memoria para optar al título de Ingeniero Civil Informático, Departamento de Informática, Universidad Técnica Federico Santa María, Diciembre, Valparaíso (Chile).

- $\quad$ Porter, M. (2003): “The Economic Performance of Regions”. Regional Studies, 37, n. 6-7, Agosto-Octubre, pp. 549-578.

• _ _ _ (1990): “La ventaja competitiva de las naciones”, Barcelona (España): Plaza \& Janés.

y Stern, S. (1998): “On Competition, Cambridge” (Ma.): Harvard Business School Press.

- $\quad$ Ramachandran, K. y Sougata, R. (1996): “Corporate Strategy Revisited: Towards Developing a Dynamic Framework”, IIMA Working Papers, 1322, Indian Institute of Management Ahmedabad.

- Rodríguez, D. (2006): "Modelos para la creación y gestión del conocimiento: una aproximación teórica”, Educar, n. 37, Departamento de Pedagogía Aplicada, Barcelona (España): Universidad Autónoma de Barcelona, pp. 25-39.

- $\quad$ Roelandt, T.J.A. y Den Hertog, P. (Eds.)(1999): “Cluster analysis and cluster-based policy making in OECDcountries”. Organización para la Cooperación y el Desarrollo Económicos (OCDE).

- $\quad$ Roos, J.; Roos, G.; Dragonetti, N.C. y Edvinsson, L. (1997): “Intellectual Capital”: Navigating the New Business Landscape. Londres (Reino Unido): Macmillan.

- Saiz, J.M. (2010): "Social Market Economy and Welfare State. Towards the Formation of a New Cybereconomy”. en Galindo, M.A. (Dir.), International Political Economy, Nueva York (Estados Unidos): Nova Science Publishers.

- _ _ (2009): “Capital intelectual, protocolo y empresa familiar”. Anuario Jurídico y Económico Escurialense, vol. 62, El Escorial (Madrid): Real Colegio Universitario "María Cristina"- Universidad Complutense de Madrid, pp. 377-388.

- _ (2006): "Capital humano, educación y transformación estructural. Una aplicación a la región euro-ártica de Suecia”, en Saiz, J. M. (Ed.), ¿Hacia dónde vas, Europa?, Madrid (España): FIEC (2ª Ed., 2007).

- _ (2004): “Claves para un nuevo mercado de trabajo. Una aplicación para la Unión Europea”, Alicante (España): Editorial Club Universitario.

• _ y De la Antonia, D. (2009): “CRM. Estrategia para la gestión”, La Coruña (España): NetBiblo.

- $\quad$ Shanker, D.J. y Astrachan, J.H. (1996): “Myths and realities: family businesses' contribution to the US economy. A framework for assessing family business statistics”. Family Business Review, vol. 9, n. 2, pp. 107-123.

• $\quad$ Storper, M. (1997): “The Regional World”, Nueva York (EE.UU.): The Guilford Press.

- Swann, G. (2002): "The Implications of Clusters. Some Reflections”, en The Cluster Conference, Manchester Business School, Abril.

- $\quad$ Uhlaner, L. (2002): “The Use of the Guttman Scale in Development of a Family Business Index”, Scales Research Reports, H200203, EIM Business and Policy Research, Ministerio de Asuntos Económicos, Zoetermeer (Holanda).

- Van Ark, B. et al (2009): “Measuring intangible capital and its contribution to economic growth in Europe”, EIB Papers, 3/2009, Luxemburgo: Banco Europeo de Inversiones.

- $\quad$ Verbeke, A.B. y Frances, S.M. (2006): “Corporate Environmental Strategy: Extending the Natural Resource-based View of the Firm”, Academy of Management Best Conference Paper, Chicago. 